\title{
Flexibilisierungspotenziale bei heterogenen Arbeitsmärkten und deren wirtschaftspolitische Implikationen
}

\author{
Bernd Fitzenberger • Olaf Hübler · Kornelius Kraft
}

Online publiziert: 27. April 2011

(C) Institut für Arbeitsmarkt- und Berufsforschung 2011

Zusammenfassung Im Jahr 2004 dominierte sowohl in der nationalen als auch in der internationalen arbeitsmarktpolitischen Debatte noch die Forderung nach mehr Flexibilität des deutschen Arbeitsmarktes (OECD 1994, 2004; Heckman 2002; SVR 2002). Demgegenüber zeigte sich 2010 die Fachwelt erstaunt über die Flexibilität des deutschen Arbeitsmarktes während der Wirtschaftskrise 2008 und 2009 (Möller 2010). Welch ein Wandel, der nicht vom Himmel gefallen ist. Flexibilitätspotenziale sind unter Berücksichtigung eines heterogenen Verhaltens arbeitsmarktrelevanter Gruppen genutzt worden. Das seit 2004 bis Ende 2010 von der DFG geförderte Forschungsschwerpunktprogramm „Flexibilisierungspotenziale bei heterogenen Arbeitsmärkten" hat Flexibilisierungspotenziale auf dem Arbeitsmarkt unter Berücksichtigung der vielfältigen Heterogenität der Arbeitsmärkte untersucht. Anliegen dieses Themenheftes ist es, einen Überblick über die im Rahmen des Programms entstandenen Forschungsbeiträge zu geben und deren wirtschaftspolitische Implikationen einer breiteren Öffentlich-

\section{B. Fitzenberger $(\bowtie)$}

Albert-Ludwigs-Universität Freiburg, Empirische

Wirtschaftsforschung und Ökonometrie, Platz der Alten

Synagoge, 79085 Freiburg, Deutschland

e-mail: fitzenbe@vwl.uni-freiburg.de

O. Hübler

Leibniz Universität Hannover, Institut für Empirische

Wirtschaftsforschung, Königsworther Platz 1, 30167 Hannover,

Deutschland

e-mail: huebler@ewifo.uni-hannover.de

K. Kraft

Technische Universität Dortmund, Wirtschafts- und

Sozialwissenschaftliche Fakultät, Vogelpothsweg 87,

44227 Dortmund, Deutschland

e-mail: kornelius.kraft@uni-dortmund.de keit zugänglich zu machen. Dieser einführende Beitrag ordnet die Arbeit des Forschungsschwerpunktprogramms im Lichte der Debatte um die Flexibilität des Arbeitsmarktes ein, beschreibt die Arbeit des Forschungsschwerpunktprogramms und gibt eine kurze Zusammenfassung der Beiträge dieses Themenheftes.

Schlüsselwörter Flexibilität · Heterogenität ·

Beschäftigung · Löhne · Arbeitsmarktinstitutionen ·

Arbeitsmarktpolitik

JEL Klassifikationen J31 · J51 · J52 - J62 · J63 • O33

Flexibility in heterogeneous labor markets and implications for economic policy

Abstract In 2004, the national and international debate on labor market performance forcefully made a case for more flexibility in the German labor market (OECD 1994, 2004; Heckman 2002; SVR 2002). In contrast, in 2010, experts were surprised by the flexibility of the German labor market during the world recession 2008 and 2009 (Möller 2010). What a change, which did not appear from nowhere. Flexibility has been increasing considering the heterogeneous behavior of labor market groups. The DFG sponsored Priority Program "Flexibility in heterogeneous labor markets" ("Flexibilisierungspotenziale bei heterogenen Arbeitsmärkten"), which ran from 2004 to 2010, analyzed potentials for flexibility in the labor market in light of multifaceted heterogeneity. The purpose of this special issue is to give an overview of research contributions in the Priority Program "Flexibility in heterogeneous labor markets" and to communicate implications for economic policy to a wider audience. This editorial puts the Priority Program into perspective in light of the flexibility debate, describes the activities of the 
Priority Program, and summarizes the contributions to the special issue.

Keywords Flexibility - Heterogeneity · Employment . Wages · Labor market institutions - Labor market policy

\section{Einleitung und Einordnung in die Flexibilisierungsdebatte}

\begin{abstract}
„Deutschland leidet unter tief greifenden strukturellen Problemen auf dem Arbeitsmarkt, ... Vor allem zwei Gründe sind für diese unbefriedigende Entwicklung verantwortlich: die Rigiditäten auf dem Arbeitsmarkt und die Lohnentwicklung. ... Der deutsche Arbeitsmarkt ist in einer desolaten Verfassung, er muss grundlegend reformiert werden." (SVR 2002, Ziffer 347 und 353)
\end{abstract}

„Apparently the German labor market system has undergone a strange mutation from a bulwark of eurosclerosis into a champion of flexibility. The specific type of German flexibility, however, does not stem from high labor turnover rates (hiring and firing), but through an unprecedented level of buffer capacity within firms. " (Möller 2010, S. 326)

Die arbeitsmarktpolitische Debatte um die mangelnde Flexibilität des deutschen Arbeitsmarktes als Ursache der persistent hohen Arbeitslosigkeit wurde intensiv zu Beginn des letzten Jahrzehntes national und international geführt (siehe unter anderem OECD 1994 und 2004; Heckman 2002; SVR 2002). Die Zahl der registrierten Arbeitslosen erreichte mit über 5 Mio. im Januar 2005 (erster Monat der Erfassung der erwerbsfähigen, vormaligen Sozialhilfeempfänger) ein Maximum. Sie ging während des Aufschwungs 2006 und 2008 deutlich zurück. Umgekehrt stieg die Beschäftigung stark an. Die Zahl der registrierten Arbeitslosen sank auf unter 3 Mio. im Oktober 2008 und stieg während der durch die Finanzmarktkrise ausgelösten Rezession 2008 und 2009 kaum an. Die Mehrheit des Sachverständigenrates (SVR 2007, Ziffer 497 und 504) konstatierte 2007, ,dass die Flexibilität und die Dynamik am Arbeitsmarkt zugenommen haben“ und „dass die Reformanstrengungen der vergangenen Jahre erste Früchte tragen". Bezugnehmend auf einen Artikel von Paul Krugman in der New York Times vom 12. November 2009 spricht Möller (2010) von einem Beschäftigungswunder in Deutschland während der jüngsten Krise. Trotz hoher Betroffenheit von der Krise, die zu einem starken Einbruch des BIP um 4,7 vH im Jahr 2009 geführt hat, ist die Arbeitslosigkeit 2009 nur unmerklich angestiegen. Möller (2010) führt dies auf die hohe betriebsinterne Flexibilität (Arbeitskräftehorten) in Deutschland zurück. Zusammen mit den Beschäftigten, die im Rahmen von betrieblichen Bündnissen vorübergehend auf Vergünstigungen und tarifvertragliche Zusagen verzichtet haben, und dem effizienten Einsatz betrieblicher und arbeitsmarktpolitischer Instrumente wie Arbeitszeitkonten und Kurzarbeit war es möglich, den durch den dramatischen Einbruch der Güternachfrage insbesondere in exportorientierten Bereichen induzierten Rückgang der Arbeitsnachfrage abzufedern. Statt zu entlassen, wurden die Zahl der Arbeitsstunden vermindert und ein Absinken der Arbeitsproduktivität geduldet. Aber nicht alle Beschäftigten haben gleichermaßen von der konzertierten Aktion profitiert. So war das Instrument der Kurzarbeit vor allem dann erfolgreich, wenn es in Verbindung mit Weiterbildungsmaßnahmen genutzt wurde und wenn nicht die Gesamtbelegschaft, sondern ca. $60 \%$ der Belegschaft von Kurzarbeit betroffen war. Längerfristig zeigen sich weniger positive Ergebnisse der Kurzarbeit in Bezug auf die Entwicklung der Beschäftigung (Hübler 2010). Dies entspricht dem Motto von Brenke et al. (2010) „Kurzarbeit Nützlich in der Krise, aber nun den Ausstieg einleiten“ und wurde auch real umgesetzt.

Für die neue Krisenfestigkeit des deutschen Arbeitsmarktes dürfen nicht nur die genannten Maßnahmen und Verhaltensweisen verantwortlich gemacht werden. Vielmehr ist auch zu berücksichtigen, dass im letzten Jahrzehnt der deutsche Arbeitsmarkt flexibler geworden ist und Arbeitsmarktrigiditäten zurückgegangen sind. Zum einen gingen nach 2002 die Lohnstückkosten in Deutschland im Vergleich zu anderen Industrieländern deutlich zurück. Gründe hierfür sind der Anstieg der Produktivität, die moderaten Tariflohnerhöhungen und der Rückgang der Spanne zwischen Effektivlöhnen und Tariflöhnen (SVR 2006, Tabelle 45). Zum anderen ist seit einigen Jahren eine starke Zunahme der Lohnungleichheit in Deutschland festzustellen (Dustmann et al. 2009; Antonczyk et al. 2010; Sengenberger 2009) und insbesondere im unteren Bereich der Lohnverteilung nimmt die Lohnungleichheit zu. Die Arbeitsmarktreformen Mitte des letzten Jahrzehnts haben augenscheinlich einen Rückgang der Arbeitsmarktrigiditäten bewirkt (SVR 2007).

Das von der DFG geförderte Forschungsschwerpunktprogramm „Flexibilisierungspotenziale bei heterogenen Arbeitsmärkten“" untersuchte im Zeitraum 2004 bis 2010 unterschiedliche arbeitsökonomische Forschungsgegenstände, meist spezifisch für Deutschland, im Hinblick auf die Flexibilität des Arbeitsmarktes. Aus dieser Arbeit ergab sich eine Vielzahl an wirtschaftspolitischen Implikationen, die im Rahmen der Beiträge dieses Themenheftes herausgearbeitet werden.

In unserem Beitrag Fitzenberger et al. (2008) nehmen wir eine Einordnung der Arbeit des DFG-Forschungsschwerpunktprogramms im Hinblick auf den Stand der arbeitsökonomischen und wirtschaftspolitischen Diskussion um die Flexibilität des Arbeitsmarktes zu Beginn des neuen Jahrtausends vor. Im Folgenden geben wir sehr kurz den Stand 
der wirtschaftspolitischen Diskussion vor und während der Laufzeit des Programms wieder.

In der sogenannten „Jobs Strategy“ forderte die OECD (1994) mehr Flexibilität verkrusteter Arbeitsmärkte, Lohnsetzungen zur Reduktion der Arbeitskosten, eine Flexibilisierung der Arbeitsmarktinstitutionen sowie eine Reduktion des Kündigungsschutzes und der Mobilitätskosten. Häufig wurde in der Debatte konstatiert, dass die berufliche Ausbildung in Ländern wie Deutschland zu spezifisch ist und dass die Humankapitalbildung gestärkt werden sollte, z. B. durch eine Erhöhung der Anzahl an Hochschulabsolventen. Ausgangspunkt dieser Forderungen war die Beobachtung persistenter Arbeitslosigkeit, die nach jeder zyklischen Krise seit Mitte der 1970er Jahre angestiegen war und im darauf folgenden Aufschwung nicht auf das Niveau vor der Krise zurückgegangen ist. In diesem Sinne wird konjunkturelle Arbeitslosigkeit persistent, da der Arbeitsmarkt keine flexible Anpassung mit neuen Jobmöglichkeiten für die Arbeitslosen zulässt. Hierzu führt der SVR (2002, Ziffer 21) aus: „Die Rigiditäten auf dem deutschen Arbeitsmarkt sind höher als in den angelsächsischen Ländern ... Allerdings zeigen sich die schädlichen Wirkungen von Rigiditäten vor allem bei exogenen Schocks wie der deutschen Vereinigung. Schließlich hat auch eine nicht beschäftigungsorientierte Lohnpolitik zur ungünstigen Beschäftigungsentwicklung beigetragen. “

Heckman (2002) betonte in seiner Analyse des deutschen Arbeitsmarktes die Gefahr einer fehlenden Wettbewerbsfähigkeit. Deutschland, so sein Argument, falle bei Spitzentechnologien zurück. Die Bedeutung spezifischen, lokalen Wissens und traditioneller Stärken sei rückläufig, da das wirtschaftliche Umfeld immer dynamischer werde. Die Arbeitsmarktinstitutionen in Deutschland werden als zu rigide angesehen, um hinreichend flexibel auf die ökonomischen Herausforderungen reagieren zu können. Da gleichzeitig die Anpassungsprobleme während der 1990er Jahre und der ersten Jahre des letzten Jahrzehnts gestiegen seien, fallen, so die Einschätzung, die volkswirtschaftlichen Anpassungskosten bei rigiden Institutionen noch stärker als ohnehin aus. In ihrer Reevaluation der ,Jobs Strategy“ betont die OECD (2004) die Notwendigkeit einer verstärkten Lohnflexibilität und einer Dezentralisierung der Lohnverhandlungen, um die Rigidität bei den Lohnvereinbarungen aufzuheben.

Trotz der moderaten Lohnentwicklung seit Mitte der 1990er Jahre war es in der Phase 2002 bis 2004 noch weitgehend offen, ob die Lohnsteigerungen, gemessen an der Produktivitätsentwicklung bei gegebener Arbeitslosigkeit, tatsächlich rückläufig waren (OECD 2004; SVR 2007, Ziffer 475-513). Zu beobachten war zudem, dass der starke Anstieg der Lohnungleichheit und eine höhere Lohnflexibilität mit einer positiven Beschäftigungsentwicklung in den OECD-Ländern einhergingen (OECD 2004). Dieser empirische Zusammenhang kann allerdings nicht zwangsläufig als ein kausaler interpretiert werden. Die relative Beschäftigungsentwicklung für junge oder ältere Arbeitnehmer ist insbesondere in Ländern, in denen die Lohnungleichheit seit den 1970er Jahren weniger stark angestiegen ist, ungünstiger verlaufen. Nach Einschätzung der OECD (2004) hat sich der Zielkonflikt zwischen positiver Beschäftigungsentwicklung und einer geringeren Einkommensungleichheit verschärft. Demgegenüber betont Horn (2011), dass die Stärkung der Wirtschaft und die Bekämpfung der Ungleichheit Hand in Hand gehen.

Die arbeitsmarktpolitischen Empfehlungen des Sachverständigenrates im Jahr 2002 lasen sich angesichts der krisenhaften Entwicklung auf dem Arbeitsmarkt wie folgt: „Den Kern eines Programms für Beschäftigung und Wachstum muss eine Reform des Arbeitsmarkts bilden, denn die derzeitigen institutionellen Rahmenbedingungen setzen gravierende Fehlanreize“ (SVR 2002, Ziffer 22). Der Sachverständigenrat betont, dass eine verbesserte Vermittlungseffizienz, wie sie die Hartz-Reformen anstrebten, keine ausreichende Antwort zur Lösung der arbeitsmarktpolitischen Probleme sei. Im Kernpunkt der Forderungen des Sachverständigenrates standen die folgenden drei Punkte: (1) Stärkung der Nachfrage nach Arbeitskräften durch Senkung des Grenzabgabensatzes für Arbeit und Lohnerhöhungen unterhalb der Zuwachsrate der Arbeitsproduktivität; (2) Senkung der Anspruchslöhne und Ausbau des Niedriglohnbereichs z. B. durch Beschränkung des Bezugsdauer von Arbeitslosengeld auf 12 Monate für alle Arbeitnehmer; (3) Herstellung von mehr Flexibilität am deutschen Arbeitsmarkt und Verbesserung der Abstimmungsmechanismen. Vorgeschlagen werden hier vor allem Maßnahmen zur Erhöhung der Flexibilität bei Tarifverträgen, indem von den Möglichkeiten der Abweichung von Regelungen der Tarifverträge Gebrauch gemacht wird. Zudem wird die Vereinbarung und Verstärkung variabler Lohnkomponenten für sinnvoll erachtet.

Inzwischen hat sich die Arbeitsmarktlage deutlich verbessert und selbst während der Krise war im April 2009 lediglich ein saison- und konjunkturbedingter Anstieg der Arbeitslosigkeit auf 3,6 Mio zu verzeichnen. Die hohe betriebsinterne Flexibilität in Deutschland hat einen weiteren Anstieg der Arbeitslosigkeit in der jüngsten Wirtschaftskrise verhindert (Möller 2010). Nach Meinung der Mehrheit des Sachverständigenrates (SVR 2007, S. 341) gibt es Anzeichen, ,dass die Reformanstrengungen der vergangenen Jahre erste Früchte tragen“. Dieser Aspekt wird auch von Schneider und Zimmermann (2010) betont. Sie plädieren für eine mutige Fortsetzung des Reformweges mit dem Ziel der Vollbeschäftigung.

Gleichzeitig mit der Verbesserung der Beschäftigungslage hat sich die Ungleichheit am Arbeitsmarkt, gemessen an der Lohnstruktur, erhöht. In den letzten zehn Jahren kam es zur Herausbildung eines starken Niedriglohnsektors. Diese Entwicklungen sind mit erheblichen Kosten verbunden (Wilkinson und Pickett 2009) und werden sehr kritisch kommentiert (Horn 2011; Sengenberger 2009). 
Sie dienen nicht zuletzt als Begründung für die seit einigen Jahren gestellte politische Forderung nach Einführung von allgemeinen Mindestlöhnen und einer stärkeren Regulierung des Arbeitsmarktes, insbesondere für den Bereich der Zeitarbeit. Die zunehmende Kluft zwischen Arm und Reich hat ihren Ursprung in ganz verschiedenen Politikund Wirtschaftsbereichen wie Staatsverschuldung, Besteuerung, Lohnsetzung, betriebliche Reorganisation, technischer Fortschritt oder Globalisierung. Sie äußert sich nicht zuletzt auch in einem Auseinanderdriften von Bevölkerungsschichten, wie zwischen gut und schlecht ausgebildeten Arbeitskräften, zwischen Rentnern und Beitragszahlern, zwischen Familien mit Kindern und ohne Kinder (Konrad und Zschäpitz 2010). Akerlof und Shiller (2009) betrachten das Problem der Ungleichheit ebenfalls mit Sorge. Hält sich Armut im Kreis von Minderheiten doch besonders hartnäckig. Die Lösung dieses Problems kann nach ihrer Auffassung nicht dem Markt überlassen werden.

Flexibilität des Arbeitsmarktes und Heterogenität der Arbeit hängen untrennbar miteinander zusammen. Im Lichte der Debatte um die mangelnde Flexibilität des deutschen Arbeitsmarktes untersuchte das zwischen 2004 und 2010 von der DFG geförderte Forschungsschwerpunktprogramm „Flexibilisierungspotenziale bei heterogenen Arbeitsmärkten", welche Potenziale für schnellere und flexiblere Anpassungen auf dem Arbeitsmarkt unter Berücksichtigung möglicher Heterogenität der Arbeitsmärkte vorhanden sind. Der Förderzeitraum ging mit der veränderten Einschätzung zur Flexibilität des deutschen Arbeitsmarktes und der generellen Verbesserung der Arbeitsmarktsituation in Deutschland einher. Dies belegt die sowohl forschungs- als auch wirtschaftspolitisch hohe Relevanz der Thematik des Schwerpunktprogramms und der daraus hervorgegangenen Ergebnisse.

In Fitzenberger et al. (2008) haben wir die Konzeption des Forschungsschwerpunktprogramms im Lichte des arbeitsökonomischen Diskussionsstandes am Ende des alten und zu Beginn des neuen Jahrtausends vorgestellt und diskutiert. Dabei wurde auch ein Überblick über Themen und Ziele der geplanten wissenschaftlichen Arbeiten gegeben. Unsere Darstellung ist von Sengenberger (2009) sehr kritisch rezipiert worden. Dies hat uns zu einer detaillierten Erwiderung veranlasst, in der wir die Ergebnisoffenheit der arbeitsökonomischen Forschung im Hinblick auf die Vorzüge konkreter Flexibilisierungsmaßnahmen betonen (Fitzenberger et al. 2009). Auf diese Diskussion im Spannungsfeld der beobachteten Veränderungen des deutschen Arbeitsmarktes hin zu einer stärkeren Flexibilisierung sei an dieser Stelle verwiesen. Ein Mehr an Flexibilität und Risiko kann angesichts von Risikoaversion der Beschäftigen durchaus problematisch sein. In diesem Band soll zum Abschluss des Schwerpunktprogramms über die wissenschaftlichen Ergebnisse einer großen Anzahl von Projekten zusammenfassend berichtet werden. Zudem geht es um die Verdeutlichung der wirtschaftspolitischen Implikationen.
Die Intention des Forschungschwerpunktprogramms war eine sorgfältige ökonomische Analyse eines Mehr oder Weniger an Flexibilität in verschiedenen Bereichen der arbeitsökonomischen Forschung (Fitzenberger et al. 2008, 2009). Die in der Vergangenheit durchaus hilfreiche Analyse des Arbeitsmarktes unter der Annahme homogener Arbeitskräfte und homogener Betriebe hat zunehmend an Erklärungskraft verloren, da sich verschiedene Teilarbeitsmärkte in einer höchst unterschiedlichen Situation befinden können. Die vielfältigen Änderungen in den Rahmenbedingungen haben sehr unterschiedliche Reaktionen auf der Anbieterund Nachfragerseite, aber auch innerhalb der Arbeitskräftegruppen und Betriebe hervorgerufen. Eine wachsende Heterogenität der Arbeitsmarktbedingungen über Qualifikationsgruppen, Berufe, Betriebe, Branchen oder Regionen hinweg ist die Folge. Als Beispiel sei genannt das gleichzeitige Auftreten von einem Nachfrageüberschuss bei hoch qualifizierten Arbeitnehmern und einem Angebotsüberschuss bei gering qualifizierten Arbeitnehmern. Offensichtlich gibt es hier Flexibilisierungsprobleme, die einen Ausgleich über Teilmärkte be- oder gar verhindern. Diese Beobachtungen führen zu neuen Herausforderungen, da den vermehrt auftretenden Starrheiten auf dem Arbeitsmarkt differenziert begegnet werden muss.

Die verschiedenen Beiträge des Themenheftes machen mit ihren ganz unterschiedlichen Fragestellungen, Methoden und Datensätzen sehr gut deutlich, dass unter dem Blickwinkel der Flexibilisierung nicht jede Maßnahme in die gleiche Richtung zielt, dass einige in der bisherigen öffentlichen und wissenschaftlichen Diskussion erwartete Effekte sich durchaus empirisch bestätigen lassen, während andere eher im Gegensatz dazu stehen (siehe hierzu Fitzenberger et al. 2008). Institutionelle Veränderungen erzielen keineswegs immer die erwarteten Wirkungen oder haben unangenehme Nebeneffekte. Die Analyse darf sich nicht auf ein einziges Wirkungsfeld beschränken. Vielmehr muss sie gerade im Hinblick auf eine wirtschaftspolitische Einschätzung den verschiedenen Dimensionen Rechnung tragen. Vor allem ist es dringend notwendig, bei den Wirkungen zwischen verschiedenen Gruppen zu unterscheiden. In Zukunft wird es noch stärker notwendig sein, das Zusammenwirken verschiedener Maßnahmen gleichzeitig zu betrachten.

Im Folgenden geben wir in Abschn. 2 einen kurzen Überblick über die Arbeit im DFG-Forschungsschwerpunktprogramm und fassen die weiteren Beiträge dieses Themenheftes in Abschn. 3 zusammen. Dieses Themenheft bildet den Abschluss der sehr erfolgreichen, auch in der Öffentlichkeit wahr genommenen gemeinsamen Arbeit im Schwerpunktprogramm. 


\section{Die Arbeit des DFG-Forschungs- schwerpunktprogramms}

Schwerpunktprogramme (SPP) übernehmen insofern eine wichtige wissenschaftspolitische Aufgabe, als sie die Bedeutung von bislang in Deutschland relativ wenig erforschten Gebieten in den Vordergrund rücken sollen. Nach unserer Einschätzung bestand zu Beginn des Programms „Flexibilisierungspotenziale bei heterogenen Arbeitsmärkten“ in Deutschland ein Nachholbedarf bei der wirtschaftswissenschaftlichen Arbeitsmarktforschung. Schwerpunktprogramme beabsichtigen grundsätzlich über die Aktivierung einer kritischen Masse und damit verbundener Synergieeffekte sowie einer Förderung über einen vergleichsweise langen Zeitraum von sechs Jahren, einen relativ zur internationalen Diskussion feststellbaren Nachholbedarf auszugleichen. Einer gut funktionierenden Kommunikation und Interaktion kommen bei der intendierten Integration unterschiedlicher Denk- und Forschungstraditionen in diesem Schwerpunktprogramm zentrale Rollen zu. Damit sollen auch bislang isoliert arbeitende Forschungsinitiativen verzahnt werden.

Wie schon ausgeführt, sollen SPP interessante, zukunftsorientierte Themen fördern. Damit ist beabsichtigt, die wissenschaftliche und wirtschaftpolitische Diskussion in Deutschland voranzubringen und den involvierten Nachwuchswissenschaftlern Gelegenheit zu bieten, Dissertationsvorhaben zu anregenden und für die deutsche Wissenschaftslandschaft aussichtsreichen Themen zu verfolgen. Einerseits wird damit der Erkenntnisstand zu zentralen Fragestellungen allgemein verbessert und andererseits können sich die Nachwuchswissenschaftler innerhalb der aktuellen wissenschaftlichen Diskussion profilieren. Gerade das von uns behandelte Gebiet der Flexibilitätsprobleme und -möglichkeiten vor dem Hintergrund der deutschen Institutionen bietet hierzu hervorragende Möglichkeiten.

Die Kommunikationsprozesse sind nach internen und externen Interaktionen zu differenzieren. Eine Umsetzung von Austausch und Kooperation innerhalb des SPP erfolgte hauptsächlich über halbjährliche Arbeitstreffen. Diese Arbeitstreffen waren gekennzeichnet durch eine rege und zahlreiche Teilnahme von Projektleitern und Mitarbeitern aus allen Projekten sowie ein intensive und konstruktive Diskussion über Vorgehensweisen und erzielte Ergebnisse.

2008 führte das Schwerpunktprogramm eine Summerschool mit internen und externen Teilnehmern durch. Ziele waren die Vermittlung neuerer Erkenntnisse aus der Arbeitsökonomik und der angewandten Ökonometrie sowie die Vorstellung und Diskussion von Forschungsergebnissen durch die Nachwuchswissenschaftler selbst.

Über jährlich stattfindende Workshops wurde der Kontakt zur internationalen Forschung ausgebaut. Die vortragenden Keynote Speakers waren weltweit führende Experten auf ihren Gebieten. Einerseits sollten diese einen Überblick zu den aktuellen Themen geben und andererseits über die Vorstellung der eigenen Forschungsergebnisse beispielhaft die Vorgehensweise innerhalb der Forschung vermitteln. Die „calls for papers“ für die Workshops fanden ein weltweites Echo und aufgrund des großen Interesses an einer Teilnahme und Präsentation konnte jeweils ein sehr attraktives Programm zusammengestellt werden.

Die Realisierung direkter Kontakte mit der aktuellen Front der Forschung und der Forschenden diente einer Vermittlung von Anregungen für die eigene Arbeit. Erfahrungen aus anderen Ländern mit ähnlichen oder auch ganz anderen Arbeitsmarktstrukturen konnten genauso diskutiert werden wie Ergebnisse unterschiedlicher Flexibilisierungsstrategien für Arbeitsmärkte in anderen Ländern. Die internationalen Tagungen dienten außerdem der Darstellung, Diskussion und Fortentwicklung der Forschungsergebnisse im Schwerpunktprogramm zusammen mit inländischen und ausländischen Experten. Speziell der Abschlussworkshop im März 2010 war auf die Präsentation unserer eigenen Ergebnisse in einem internationalen Rahmen ausgerichtet.

Ein weiterer Aspekt der Diskussion und Verbreitung der Ergebnisse des Schwerpunktprogramms war die Unterstützung von Konferenzbesuchen unserer Mitglieder. Die Ergebnisse unserer Arbeit wurden auf führenden nationalen und internationalen Tagungen präsentiert. Durch die Teilnahme an den internationalen Kongressen bot sich eine über den Schwerpunkt hinausgehende Plattform zur Diskussion und es konnten interessante Anstöße für eine Weiterentwicklung des Bereiches Arbeitsmarktforschung in Deutschland aufgenommen werden. Das SPP förderte weiterhin die Teilnahme an zusätzlichen Summer Schools und längere Auslandsaufenthalte unserer jüngeren Mitglieder.

\section{Beiträge des Themenheftes}

Neben diesem einführenden Artikel enthält das Themenheft 20 weitere Beiträge, die mindestens einem der folgenden drei Themenbereiche zuzuordnen sind: (1) Lohnniveau, Lohnstrukturen und Arbeitsmarkterfolg, (2) Beschäftigung und Übergänge im Arbeitsmarkt und (3) Arbeitsmarktinstitutionen und arbeitsmarktpolitische Maßnahmen. Im Folgenden fassen wir die Inhalte der Beiträge nach diesen Themengebieten zusammen. Die meisten Beiträge nehmen jedoch auf zwei oder gar auf alle drei Schwerpunkte Bezug. Das macht deutlich, dass in den Einzelprojekten nicht eindimensional geforscht wurde, sondern in jedem Fall versucht wurde, der Komplexität der Thematik gerecht zu werden und Verknüpfungen zu anderen Projekten des Schwerpunktprogramms herzustellen. Dazu hat der rege Gedankenaustausch über inhaltliche Problemstellungen, Daten, Methoden und Ergebnisse in den Arbeitstreffen und Workshops erheblich beigetragen. Dieses Themenheft gibt einen Überblick über die erzielten Forschungsergebnisse der beteiligten Wissenschaftlerinnen und Wissenschaftler im Rahmen 
des SPP und arbeitet die wirtschaftspolitischen Implikationen heraus.

Die wirtschafts- und unternehmenspolitischen Schlussfolgerungen fallen themenbezogen recht unterschiedlich aus. Gemeinsam ist jedoch allen, wie die empirischen Untersuchungen zeigen, dass Maßnahmen, die nicht gruppenspezifisch, sondern undifferenziert ausgerichtet sind, weniger positiv in ihren Wirkungen eingeschätzt werden. Historische Gegebenheiten und institutionelle Besonderheiten, die in der Vergangenheit in der ökonomischen Analyse eher einen geringen Stellenwert hatten, erweisen sich als wichtige Katalysatoren dafür, ob und in welchem Umfang Arbeitsmarktpolitik erfolgreich ist. Sie besitzen einen erheblichen Beharrungsgrad, der in einigen Bereichen wünschenswert ist, der in anderen Zusammenhängen jedoch dafür sorgt, dass notwendige Veränderungen erst mit Verzögerung wirksam werden. Erfolg versprechend sind solche Maßnahmen und Regelungen, die von der Mehrheit der Marktakteure getragen werden, die mit ihren Zielen vereinbar sind. Die empirischen Untersuchungen haben aber auch deutlich gemacht, dass nicht jede gesetzliche oder vertraglich vereinbarte Regelung eine schnelle und flexible Anpassung an veränderte Rahmenbedingungen verhindert, wie dies häufig in Politik, Medien und Öffentlichkeit vermutet wird.

\subsection{Lohnniveau, Lohnstrukturen und Arbeitsmarkterfolg}

Antonczyk et al. (2011) untersuchen die Entwicklung der Lohnungleichheit in Deutschland seit den 1990er Jahren. Als mögliche Ursachen für Veränderungen werden die abnehmende Tarifbindung und die Polarisierungshypothese (Beschäftigung von Hoch- und Niedrigqualifizierten steigt relativ zur Beschäftigung von Personen mit mittlerem Qualifikationsniveau) aufgenommen. Die Lohnungleichheit hat in dem untersuchten Zeitraum stark zugenommen. Sie lässt sich jedoch nur zu einem geringen Teil aus der Abnahme der Tarifbindung erklären. Wichtiger sind Veränderungen der Lohnunterschiede zwischen und innerhalb der Wirtschaftszweige. Es lässt sich seit den 1990er Jahren für Deutschland keine Tendenz zu einer zunehmenden Polarisierung der Entlohnung erkennen. Der Anstieg der Arbeitsnachfrage für einfache Nichtroutinetätigkeiten ist bisher nicht mit Lohngewinnen im Niedriglohnsektor einhergegangen.

Der Beitrag von Orlowski und Riphahn (2011) fasst die Ergebnisse einer Reihe von Untersuchungen zusammen, die sich auf Basis der Daten des deutschen Sozio-ökonomischen Panels (SOEP) mit der Lohnstruktur auf deutschen Arbeitsmärkten beschäftigten. Die Analysen untersuchen die Lohneffekte der Indikatoren von betriebsspezifischer und allgemeiner Arbeitsmarkterfahrung (Tenure and Experience). Um der möglichen Endogenität dieser Variablen Rechnung zu tragen, nutzen die Autoren neben Kleinstquadrateschätzern auch die von Altonji und Shakotko (1987) und von Topel (1991) verwendeten Schätzverfahren. Der Fokus liegt auf der Analyse möglicher Heterogenitäten in den Lohnstrukturen im privaten und im öffentlichen Sektor, in Ostund in Westdeutschland, für Männer und Frauen sowie bei Arbeitgebern in großen und kleinen Unternehmen. Zu den interessantesten Ergebnissen gehören die deutlichen Unterschiede zwischen Ost und West sowie zwischen großen und kleinen Unternehmen.

Seit 2009 bietet das Bundesministerium für Familie, Senioren, Frauen und Jugend (BMFSFJ) mit dem Analyseinstrument Logib-D einen freiwilligen statistischen Selbsttest auf Entgeltgleichheit in Unternehmen an. Beblo et al. (2011) passen das im Rahmen ihres Forschungsprojektes innerhalb des SPP entwickelte Schätzmodell zur Bestimmung innerbetrieblicher Entgeltunterschiede zwischen weiblichen und männlichen Beschäftigten an den Lohnrechner Logib-D an und bestimmen auf Basis des repräsentativen ArbeitgeberArbeitnehmer-Datensatzes des Instituts für Arbeitsmarktund Berufsforschung (LIAB) die geschlechtsspezifischen Entgeltlücken in deutschen Betrieben. Die Analyse bietet eine empirische Grundlage zur Einordnung der sich aus Logib-D ergebenden Einzelbefunde und zeigt, dass in der Mehrheit der deutschen Betriebe (81 Prozent im Westen und 64 Prozent im Osten) erhebliche Entgeltunterschiede zwischen den Geschlechtern bestehen, die sich nicht durch Bildungs- oder Erfahrungsunterschiede der Beschäftigten erklären lassen. Dass der innerbetriebliche Verdienstabstand zwischen Männern und Frauen stark variiert, scheint zum Teil auf systematische Unterschiede zwischen den Betrieben zurückzugehen. Daher charakterisieren Beblo et al. (2011) diejenigen Betriebe, welche einen Verdienstabstand von 5 Prozent über- oder unterschreiten anhand ausgewählter Merkmale. Die Ergebnisse erlauben neue Einblicke in Lohnstrukturen und Lohnbildungsprozesse in Betrieben und helfen, Ansatzpunkte für die Verringerung von Entgeltungleichheit aufzuzeigen.

Lohnungleichheit zwischen verschiedenen Arbeitsmarktakteuren und Gruppen, aber auch eine Veränderung der Lohnstruktur wird durch eine ganze Reihe von Faktoren hervorgerufen. Bevorzugtes Untersuchungsfeld sind geschlechts-, sektor- und qualifikationsspezifische Unterschiede. Individuelle, betriebliche und institutionelle Bestimmungsgründe werden zur Erklärung herangezogen. In neuerer Zeit ist das Augenmerk besonders auch auf die Spreizung der Einkommen im unteren und im oberen Teil der Lohnverteilung gerichtet. Auf wesentliche Teile dieser Thematik sind das Projekt und der daraus folgende Beitrag von Möller und König (2011) ausgerichtet. Die empirischen Analysen gehen folgenden Fragen nach: Inwieweit unterscheidet sich die Lohnstruktur in Ländern mit hoher und niedriger Lohnflexibilität? Haben Mindestlöhne einen wesentlichen Einfluss auf die Lohnspreizung? Sind die Lohndifferenzen zwischen Frauen und Männern regional unterschiedlich? Es zeigt sich, dass in Deutschland, einem Land, 
das lange Zeit als inflexibel auf dem Arbeitsmarkt galt, die Ungleichheit seit Mitte der 1990er Jahre merklich zugenommen hat. Für das Baugewerbe, für das erstmals die Wirkung von Mindestlöhnen analysiert wurde, ergab sich, dass im unteren Lohnbereich eine Lohnstauchung eingetreten ist und daraus in Ostdeutschland, aber nicht in Westdeutschland ein statistisch gesicherter Beschäftigungsrückgang resultierte. Zudem ergab sich, dass in ländlichen Regionen der Einkommensnachteil der Frauen gegenüber Männern erheblich größer ist als in Großstädten.

Die Studie von Bellmann (2011) untersucht die Auswirkungen organisatorischer Änderungen auf die betriebliche Entlohnung und Beschäftigung. $\mathrm{Zu}$ erwarten ist, dass organisatorische Neuerungen wie Abbau von Hierarchieebenen, Job Rotation oder Gruppenarbeit tendenziell die Anforderungen an die Beschäftigten erhöhen. Daher könnten von solchen Veränderungen zwar qualifizierte, jedoch nicht unqualifizierte Arbeitnehmer profitieren. Grundlage der empirischen Untersuchung bildet ein aus dem IABBetriebspanel und den Sozialversicherungsdaten erstellter Linked-Employer-Employee-Datensatz. Bellmann findet aufgrund von Nachfrageschätzungen keine Hinweise auf einen „skill-bias“ des organisatorischen Wandels. Löhne und Beschäftigungsstabilität werden jedoch eher bei den qualifizierten Arbeitnehmern positiv beeinflusst. Als wirtschaftspolitische Konsequenz empfiehlt er vor allem Weiterbildungsförderung der Un- und Angelernten on-the-job.

Kraft (2011) widmet sich ebenfalls den Auswirkungen organisatorischer Änderungen. Allerdings geht es hier um die Einführung von Gewinn- und Kapitalbeteiligung sowie um vermehrte Mitsprache der Belegschaft. Diese Änderungen können von den Unternehmen in Absprache mit den Beschäftigten und deren Vertretern erfolgen oder aufgrund gesetzlicher Vorgaben notwendig werden. Die Untersuchungen zielen darauf $a b$, die Wirkungen auf den Unternehmenserfolg zu erfassen. Dabei steht die empirische Analyse vor dem methodischen Problem, dass beobachtete Merkmale und unbeobachtete Einflüsse sowohl für die Entscheidung, eine der genannten Organisationsänderungen durchzuführen, als auch für die Variable, die den Unternehmenserfolg misst, von Bedeutung sind. Diesem Tatbestand wird durch spezielle ökonometrische Ansätze Rechnung getragen. Es zeigt sich, dass viele freiwillig eingeführte Maßnahmen die erwarteten positiven Auswirkungen haben, dass für den Erfolg aber auch ganz spezielle Unternehmensmerkmale erfüllt sein müssen. Selektion aufgrund von Heterogenität scheint auch hier wie in anderen Bereichen des Arbeitsmarktes eine maßgebliche Rolle für den Erfolg zu spielen.

Wirtschaftlicher Erfolg hängt von einer ganzen Reihe individueller und betrieblicher Merkmale ab. Einem bisher wenig untersuchten Aspekt widmet sich der Beitrag von Rinne et al. (2011). Es geht um die Bedeutung ethnischer
Vielfalt. Zwar gibt es viele Untersuchungen, die auf Unterschiede zwischen Einheimischen und Migranten abstellen. Eine solch einfache Dichotomie greift in vielen Fällen aber zu kurz. Innerhalb der Gruppe der Immigranten sowie zwischen Einwanderern der ersten und zweiten Generation gibt es zentrale Unterschiede. Der Beitrag geht der Frage nach, ob ethnische Vielfalt aus ökonomischer Sicht eher ein Problem oder eine Chance darstellt. Für die Beurteilung spielt der Grad der Identifikation mit der Kultur und der Gesellschaft des Aufnahme- und des Herkunftslandes eine wesentliche Rolle. Empirische Untersuchungen werden mit Hilfe des Sozio-ökonomischen Panels und des IZAEvaluationsdatensatzes durchgeführt. Es zeigt sich, dass bei der Gruppe der türkischen Einwanderer die Integration bisher weniger als bei anderen ethnischen Gruppen gelungen ist, dass aber ein positiver Trend festgestellt wird. Deutliche Unterschiede lassen sich zwischen arbeitslosen Einheimischen und Einwanderern der zweiten Generation ausmachen. Das gilt insbesondere hinsichtlich der Risikopräferenzen.

\subsection{Beschäftigung und Übergänge im Arbeitsmarkt}

Ziel des Beitrages von Gerlach et al. (2011) ist es, einen Überblick über ihre neueren Forschungsergebnisse zum Spannungsfeld der Beschäftigung zwischen Mobilität und Stabilität - aus individueller und betrieblicher Sicht sowie unter Berücksichtigung institutioneller Einflüsse - zu geben. Die vorgestellten Arbeiten gehen stärker als die bisherige Literatur auf die Interaktionen zwischen Individuum und Betrieb ein. Die Ergebnisse lassen sich vier Projektschwerpunkten zuordnen. Erstens identifiziert eine Anzahl von Studien individuelle, betriebliche und gesamtwirtschaftliche Determinanten der Beschäftigungsstabilität. Zweitens wird die Bedeutung von Löhnen und Zufriedenheit auf Arbeitsplatzwechsel untersucht. Die Befunde zeigen unter anderem, dass höhere Arbeitsplatzsicherheit und höhere Löhne in Deutschland Hand in Hand gehen. Allerdings lassen sich auch Hinweise auf kompensierende Lohndifferentiale finden, soweit es unbeobachtete Merkmale betrifft. Ein dritter Schwerpunkt analysiert die Bedeutung von Institutionen für die Beschäftigungsstabilität. Die Ergebnisse weisen darauf hin, dass die Beschäftigung in Betrieben, die Tarifverträge anwenden, stabiler ausfällt, während betriebliche Bündnisse zumindest mittelfristig keine positiven Beschäftigungswirkungen hatten. Schließlich ging ein vierter Projektteil der Wirkung von Eingliederungszuschüssen auf Löhne und Beschäftigungsdauer nach. Dabei zeigte sich, dass geförderte Beschäftigungsverhältnisse bei ungefähr gleich hohen Einstiegslöhnen länger andauern.

Boockmann und Steffes (2011) präsentieren empirische Ergebnisse zur Beschäftigungsdynamik in Deutschland. Untersucht werden hierbei die Beschäftigungsdauer und die 
Abgangswahrscheinlichkeit auf der Ebene der individuellen Arbeitnehmer sowie deren Determinanten. Die Autoren untersuchen weiterhin Fluktuationsraten auf der Betriebsebene und die Auswirkungen von konjunkturellen Schwankungen. Effekte von institutionellen Faktoren werden über den Kündigungsschutz und die Existenz eines Betriebsrates mit aufgenommen. Beide Faktoren beeinflussen die Beschäftigungsstabilität erheblich. In einer weiteren Studie werden Karriereverläufe von Frauen nach der Geburt ihres ersten Kindes betrachtet. Hierbei ergeben sich deutliche Unterschiede in Abhängigkeit von der Art der Tätigkeit der Frauen im Unternehmen. Frauen in Leitungsfunktionen weisen eine höhere Beschäftigungsstabilität auf.

Der Beitrag von Beckmann et al. (2011) diskutiert Möglichkeiten für eine unternehmensinterne flexible Reaktion auf die stetig ansteigenden Anforderungen des Arbeitsmarktgeschehens. Im Zentrum stehen dabei zwei Personengruppen: Frauen und ältere Arbeitnehmer. Zwei unternehmerische Handlungsfelder, die potenziell für Flexibilisierungsstrategien aussichtsreich zu sein scheinen, werden genauer analysiert: Weiterbildung und die Nutzung flexibler Beschäftigungsverhältnisse in Form von Leiharbeit und befristeten Verträgen. Unter Verwendung von Unternehmensund Individualdatensätzen wird nachgewiesen, dass die Veränderungen des technologischen und organisatorischen Wandels einerseits Frauen und jüngeren Arbeitnehmern nützen, aber andererseits Männern und älteren Arbeitnehmern eher schaden. Weiterbildung der Beschäftigten wie auch befristete Beschäftigungsverhältnisse und Leiharbeit erweisen sich sowohl für Unternehmen wie auch für die Beschäftigten als positiv.

Suchverhalten von Arbeitslosen ist ein zentraler Untersuchungsgegenstand der Arbeitsmarktforschung. Reservationslohn und Suchanstrengungen bestimmen nach klassischer Auffassung vor allem den Erfolg der Suche. Caliendo und Uhlendorff (2011) finden aufgrund ihrer empirischen Analysen mit Hilfe des IZA-Evaluationsdatensatzes heraus, dass nicht nur diese Suchdeterminanten und institutionelle Rahmenbedingungen für einen erfolgreichen Übergang von der Erwerbslosigkeit in Beschäftigung verantwortlich sind, sondern auch Persönlichkeitsmerkmale und individuelle Erwartungen. Personen mit kürzeren Anspruchsrechten auf Arbeitslosengeld verlassen schneller als andere den $\mathrm{Zu}-$ stand der Arbeitslosigkeit. Arbeitskräfte, die glauben, durch eigenes Handeln positiv Einfluss auf die Entwicklung ihres Berufslebens nehmen zu können, weisen eine höhere Suchintensität und einen höheren Anspruchslohn auf als andere. Arbeitslose senken dann ihren Anspruchslohn und erhöhen die Suchanstrengungen, wenn sie davon ausgehen, mit hoher Wahrscheinlichkeit an arbeitsmarktpolitischen Maßnahmen teilnehmen zu können.

Das Hauptziel des Beitrages von Fedorets und SpitzOener (2011) ist es, die Mobilität von Erwerbstätigen zu untersuchen, die ihre Ausbildung im Rahmen des dualen Ausbildungssystems absolviert haben. Grundlage der Analyse ist der Humankapital- und Suchtheorieansatz sowie die Annahme variierender Berufsinhalte über die Zeit. Mit Hilfe von Regressionsanalysen wird festgestellt, dass Berufsmobilität positiv mit dem individuellen Lohn korreliert ist. Die Höhe der Korrelation ist nicht zuletzt durch die inhaltliche Ähnlichkeit des Ausbildungs- und Erwerbsberufs sowie durch die Freiwilligkeit des Berufswechsels bestimmt. Die Ähnlichkeit des Ausbildungs- und Erwerbsberufs wird anhand eines tätigkeitsbasierten Ansatzes gemessen. Der Vergleich der Berufswechsel in West- und Ostdeutschland nach der Wiedervereinigung 1990 führt zu einer wichtigen Erkenntnis: Die meisten Berufswechsel in Ostdeutschland nach 1990 brachten hohe Lohneinbußen mit sich. Dieser negative Effekt der Anpassung der Beschäftigungsstruktur ist nachhaltig und auch nach ca. 10 Jahren noch beobachtbar. Die empirischen Ergebnisse deuten darauf hin, dass das während der Ausbildung akkumulierte Humankapital nachhaltig zwischen unterschiedlichen Berufen transferierbar ist.

Flexibilität auf dem Arbeitsmarkt heißt vor allem auch eine Anpassung der Beschäftigung an die Güternachfrage. Dies können Anpassungen in qualifikatorischer, sektoraler oder regionaler Hinsicht sein. Unterbleiben Reaktionen dieser Art, dann kann daraus ein verringertes Wachstum resultieren und interregionale Ungleichheiten bleiben möglicherweise bestehen. In Deutschland herrschte im internationalen Vergleich lange Zeit nur eine schwache räumliche Mobilität vor. Der Beitrag von Arntz (2011) setzt sich mit der Frage auseinander, ob und inwieweit dafür institutionelle Ursachen oder arbeitsmarktpolitische Maßnahmen verantwortlich sind. Im Rahmen eines Forschungsprojektes der Autorin wurden aufgrund empirischer Untersuchungen drei zentrale Ergebnisse herausgearbeitet, die im Detail in fünf Arbeitspapieren oder Veröffentlichungen dargelegt sind. Erstens hat sich gezeigt, dass unqualifizierte Arbeitslose kaum auf unterschiedliche Arbeitsmarktbedingungen in den Regionen reagieren, also kaum Wanderungsbewegungen induziert werden. Zweitens kann ein regionales Angebot an aktiver Arbeitsmarktpolitik die Mobilitätslücke zwischen qualifizierten und weniger qualifizierten Arbeitskräften nicht erklären. Drittens führt eine großzügige Arbeitslosenunterstützung zu verringerter Mobilitätsbereitschaft.

\subsection{Arbeitsmarktinstitutionen und arbeitsmarktpolitische Maßnahmen}

In den meisten Fällen werden Arbeitsmärkte unabhängig von Gütermärkten untersucht. Göddeke et al. (2011) verbinden beide Märkte und berücksichtigen, dass es unvollkommenen Wettbewerb auf Arbeitsmärkten in der Form von Gewerkschaften und ebenso Marktmacht auf Gütermärkten über Oligopole geben kann. Die Wechselwirkungen zwischen den Märkten werden unter Berücksichtigung 
aktueller Entwicklungen auf dem deutschen Arbeitsmarkt analysiert. Dies führt zu interessanten Ergebnissen. Beispielsweise können Mindestlöhne zur Abwehr von Marktzutritten dienen und dies wiederum kann die Beschäftigung stark beeinflussen. Gewinnbeteiligungssysteme können die gesamtwirtschaftliche Wohlfahrt erhöhen. Bei Existenz von Spartengewerkschaften sind unternehmensindividuelle Verhandlungen den Branchenverhandlungen wohlfahrtstheoretisch überlegen. Verhandeln die Spartengewerkschaften nicht separat voneinander, so ergibt sich eine überlegene Lösung. Die Berücksichtigung von internationalem Wettbewerb kann Ergebnisse bezüglich der Wohlfahrtswirkungen von Flächentarifverträgen stark verändern.

Der Artikel von Gürtzgen (2011) stellt empirische Ergebnisse hinsichtlich der Wirkung der Tarifbindung auf den $\mathrm{Zu}-$ sammenhang zwischen betriebsspezifischen Gegebenheiten und der Lohnhöhe vor. Es erweist sich, dass bei Vorliegen einer Bindung an einen Flächentarifvertrag die Löhne wenig Bezug zu der betriebsspezifischen Ertragslage aufweisen. Dies wird hauptsächlich durch eine geringe Anpassung an kurzfristige Schocks verursacht. Werden hingegen langfristige Veränderungen betrachtet, so findet sich insbesondere für die größeren Betriebe eine Reaktion der Löhne. Bei Flächentarifverträgen wird in heterogenen Branchen auf die Probleme der schwächeren Firmen insoweit eingegangen, als die Tarifabschlüsse bei einer zentralen Lohnfestlegung niedriger ausfallen.

Noch bis vor wenigen Jahren wurden die deutschen Arbeitsmarktinstitutionen immer wieder kritisiert, dass sie bei zunehmendem internationalem Wettbewerb zu wenige Möglichkeiten zur Anpassung von Lohnstruktur und Beschäftigung böten. Dabei zeigt sich bei genauerer Betrachtung, dass seit spätestens Mitte der 1990er Jahre ein Reformprozess in Gang gekommen ist, welcher das formal starre System der Lohnsetzung zunehmend flexibilisiert und dezentralisiert hat. Der Beitrag von Brändle et al. (2011) fasst die Entwicklungen der in Tarifverträgen verankerten Flexibilisierungsmöglichkeiten zusammen. Diese haben in Form von Öffnungsklauseln stark zugenommen. Der Beitrag gibt zunächst einen Überblick über die bisherige Forschung im Bereich der Flexibilisierung von tariflichen Vereinbarungen und zeigt die bestehenden Lücken auf. Auf Basis des IAWÖffnungsklauseldatensatzes, eines im Projekt selbst erstellten Datensatzes, ist es möglich, das Ausmaß der tariflichen Öffnung zu quantifizieren. Auf dieser Basis werden die Determinanten der Einführung von Öffnungsklauseln auf Branchenebene sowie deren betriebliche Anwendung auf Firmenebene ermittelt. Der letzte Teil des Beitrags widmet sich den wesentlichen Zusammenhängen zwischen tarifvertraglicher Öffnung und der betrieblichen Entwicklung mit besonderem Blick auf die Lohnstruktur und das Beschäftigungswachstum. Im Ergebnis zeigt sich, dass Tariföffnungsklauseln im Zeitverlauf zugenommen haben und dass mit ihnen eine Spreizung der Lohnstruktur sowie eine Steigerung des Beschäftigungswachstums verbunden sind.

Im Fokus der Arbeit von Koller et al. (2011) stehen die Auswirkungen von Festlegungen des deutschen Sozialrechts. Kleine Unternehmen werden von den Regelungen häufig ausgenommen, da die Umsetzung in der Regel mit zusätzlichen Kosten verbunden ist. D. h., die gesetzlichen Eingriffe werden üblicherweise erst ab dem Erreichen einer bestimmten Beschäftigungsgrenze relevant. Wenn nun das Überschreiten der Schwellenwerte tatsächlich mit zusätzlichen Kosten verbunden ist, dann könnte sich ein negativer Effekt hinsichtlich der Beschäftigungsausweitung bei denjenigen Firmen feststellen lassen, die sich in der Nähe der Grenzwerte befinden. Hierfür kann jedoch in Bezug auf Regelungen zur Beschäftigung von Schwerbehinderten sowie auf durch das Betriebsverfassungsgesetz festgelegte Vorgaben kein statistisch gesicherter empirischer Beleg gefunden werden.

Der Beitrag von Müller und Steiner (2011) untersucht die Beschäftigungswirkungen von Lohnsubventionen und eines Mindestlohnes für Deutschland. Studien zum Mindestlohn im Baugewerbe und Simulationen zu einem allgemeinen Mindestlohn weisen einhellig auf Beschäftigungsverluste durch einen gesetzlichen Mindestlohn für die Bundesrepublik hin. Die Größenordnung hängt vom Mindestlohnniveau, den Gütermarktreaktionen und der Reichweite des Mindestlohnes (Einschluss Auszubildender, geringfügig Beschäftigter) ab. Bei einem Mindestlohn von 7,50€ pro Stunde und elastischer Güternachfrage wird ein Gesamtverlust von ca. 220.000, insbesondere für geringfügig Beschäftigten, geschätzt. Evaluationen bestehender Lohnsubventionen finden nur geringe Effekte auf den Anreiz der Arbeitsaufnahme. Im Beitrag wird alternativ der aufkommensneutral gestaltete „Beschäftigungsbonus“, der geringe Stundenlöhne und nicht niedrige Erwerbseinkommen subventioniert, betrachtet. Dieser würde ein zusätzliches Arbeitsangebot von ca. 80.000 Personen oder - anders ausgedrückt - ein zusätzliches Stundenangebot von etwa 420.000 Vollzeitäquivalenten induzieren. In Kombination mit einem allgemeinen Mindestlohn wären arbeitnehmerseitige Lohnsubventionen ineffektiv. Die Simulationsergebnisse zeigen, dass in diesem Fall arbeitgeberseitige Lohnsubventionen die infolge des Mindestlohnes gestiegenen Arbeitskosten kompensieren und Beschäftigungsverluste im Niedriglohnbereich teilweise verringern können.

Sozialhilfe hat eine ähnliche Wirkung wie ein Mindestlohn und es werden negative Anreize auf das Arbeitsangebot erwartet. Um diese negativen Effekte zu vermeiden oder zumindest abzumildern, lässt sich nach amerikanischem Vorbild das Konzept des Workfare einführen, indem der Transferanspruch an die Verpflichtung, eine Erwerbstätigkeit auszuüben, gekoppelt wird. Hierzu gab es für Deutschland bisher keine empirischen Untersuchungen über deren Effekte. 
Schneider et al. (2011) legen erstmals Resultate aufgrund eines Pilotprojektes eines Berliner Sozialamtes vor. Geplant war, das Projekt so zu gestalten, dass es sich um ein echtes soziales Experiment handelt. Tatsächlich traten jedoch Selektionseffekte auf, so dass die Autoren mit geeigneten ökonometrischen Methoden korrigieren mussten. Die auf diesem Weg gewonnenen Befunde zeigen, dass die Beschäftigungswahrscheinlichkeit mittels Workfare zwar gegenüber reiner Sozialhilfe steigt, dass dies Ergebnis allerdings aufgrund der geringen Fallzahl statistisch nicht gesichert ist. Bei kleinen Fallzahlen spricht einiges dafür, echte Experimente durchzuführen.

Puhani und Sonderhof (2011) illustrieren die Evaluation sozialpolitischer Regeländerungen anhand von zwei Reformen, die Eingriffe in die Rechte der Arbeitgeber und Arbeitnehmer vornahmen. Kausale Effekte der Reformen werden anhand von Kontrollgruppen-Ansätzen ermittelt (hier Differenz-von-Differenzen-Schätzungen). Die Ergebnisse zeigen, dass Eingriffe des Staates in den Arbeitsmarkt neben den gewollten positiven unterstützenden Effekten - im einen Fall für Kranke, im anderen Fall für Eltern - auch negative Effekte haben. Die Verpflichtung des Arbeitgebers, einem abwesenden Arbeitnehmer 100 Prozent statt 80 Prozent des Lohnes zu bezahlen, erhöht die durchschnittlichen Abwesenheitstage vom Arbeitplatz von ca. 6 auf ca. 8 Tage (Abweichungen je nach Spezifikation; einige Schätzungen liefern sogar noch etwas größere Effekte), ohne das subjektive Gesundheitsempfinden der Arbeitnehmer nachweislich negativ zu beeinträchtigen. Die Verpflichtung des Arbeitgebers, Eltern bis zu 3 Jahre den Arbeitsplatz auch bei Abwesenheit zu garantieren, reduziert für junge Frauen den Umfang der Weiterbildung, die der Arbeitgeber anordnet.

\section{Schluss und Ausblick}

Das Forschungsschwerpunktprogramm „Flexibilisierungspotenziale bei heterogenen Arbeitsmärkten" hatte das Ziel, über die Laufzeit von 2004 bis 2010 und die Zusammenführung von interessanten Projekten in Deutschland die arbeitsökonomische Forschung auf internationales Niveau zu bringen. Wir sind der Meinung, dass uns dies auch gelungen ist. Wir konnten die wichtigsten Arbeitsmarktwissenschaftler(innen) für eine Mitwirkung gewinnen und einen sehr aktiven Diskussions- und Austauschprozess anstoßen. Das SPP hat wertvolle Projekte unterstützt und so zu einer Vielzahl von Dissertationen, Habilitationen und nicht zuletzt anspruchsvollen Forschungsarbeiten geführt. Die Anwendung von modernen ökonometrischen Methoden auf die Untersuchung von Flexibilisierungsabläufen im Kontext institutioneller Rahmenbedingungen ist sowohl wissenschaftlich als auch wirtschaftpolitisch äußerst verdienstvoll. Unserer Meinung nach unterstreichen die Beiträge zu diesem Band diese Aussage eindrücklich.
Die arbeitsökonomische Forschung ist jedoch nicht in der Flexibilisierungsdebatte stehen geblieben (Ashenfelter und Card 2011). Seit der Jahrtausendwende hat sich die arbeitsökonomische Forschung deutlich weiterentwickelt und die arbeitsmarktpolitischen Themen haben sich im Lichte der in Deutschland stark zugenommenen Ungleichheit, der demographischen Veränderungen und der sich aus der Wirtschaftskrise 2008-2009 neu stellenden Herausforderungen gewandelt. Gleichzeitig sind bedeutende Fortschritte im methodischen Bereich (insbesondere im Hinblick auf Kausalanalysen, in der Umsetzung von strukturellen Modellen und in der Durchführung und Analyse von Laborexperimenten) gemacht worden, wobei die arbeitsökonomische Forschung bei diesen Themen eine Führungsrolle im Bereich der angewandten Wirtschaftsforschung einnimmt (Ashenfelter und Card 2011). Schließlich hat sich das Themenfeld der arbeitsökonomischen Forschung stark ausgeweitet. Einerseits werden verstärkt bildungsökonomische Themen und Themen der frühkindlichen Entwicklung im Zusammenhang mit Arbeitsmarktergebnissen untersucht. Andererseits wird der $\mathrm{Zu}$ sammenhang zwischen Wirkungen auf dem Arbeitsmarkt und denen in anderen Lebensbereichen, wie z. B. bei der individuellen Gesundheit, immer intensiver erforscht.

Danksagung Wir danken der DFG für die finanzielle Förderung des Schwerpunktprogramms 1169, den beteiligten Wissenschaftlern an diesem Programm für ihre Arbeit und vor allem Marie Paul (geb. Waller) und Benedikt Lang für ihren engagierten Einsatz im Koordinationsprojekt.

\section{Anhang A: Liste der Forschungsprojekte im DFG-Forschungsschwerpunktprogramm}

Projektnummer: $P 1$

Projektleiter: Beckmann, M. und Schauenberg, $B$.

Betriebliche Personalpolitik bei technologischem und organisatorischem Wandel

Projektlaufzeit: 6 Jahre (2004-2010)

Projektnummer: $P 2$

Projektleiter: Bellmann, L. und Kleinhenz, G.

Beschäftigungs- und Lohnwirkungen von betrieblichen Reorganisationsprozessen

Projektlaufzeit: 2 Jahre (2004-2006)

Projektnummer: P3

Projektleiter: Berninghaus, S., Güth, W. und Ramser, H.J. Inflexibilitäten und die Rolle strategischer Interaktion bei der Festlegung der Lohn- und Beschäftigungspolitik von Firmen, die als Nachfrager auf heterogenen Arbeitsmärkten auftreten

Projektlaufzeit: 6 Jahre (2004-2010)

Projektnummer: $P 4$

Projektleiter: Burda, M.C. und Fitzenberger, B. 
Die Rolle der Gewerkschaften bei der Entstehung und Veränderung von Varianz und höheren Momenten der bedingten und unbedingten Lohnverteilung

Projektlaufzeit: 6 Jahre (2004-2010)

Projektnummer: P5

Projektleiter: Arntz, M. und Entorf, $H$.

Flexibilisierungspotenziale heterogener regionaler Arbeitsmärkte durch räumliche Mobilität

Projektlaufzeit: 6 Jahre (2004-01/2011 mit drei Monaten Unterbrechung)

Projektnummer: $P 6$

Projektleiter: Fitzenberger, B., Dustmann, C., Bender, S./ Rässler, S. und Schönberg, $U$.

Tarifgebundenheit, Lohnbildung und Ausbildungsanreize

Projektlaufzeit: 6 Jahre (2004-2010)

Projektnummer: $P 7$

Projektleiter: Boockmann, B. und Franz, W.

Beschäftigungsdynamik und heterogene Arbeitsverhältnisse: Untersuchungen zur Arbeitsmarktsegmentierung auf betrieblicher Ebene

Projektlaufzeit: 6 Jahre (2004-2010)

Projektnummer: $P 8$

Projektleiter: Goerke, L.

Tatsächliche Flexibilität: Arbeitsmarktregulierungen und ihre Umgehung

Projektlaufzeit: 6 Jahre (2004-2011 mit Unterbrechung)

Projektnummer: $P 9$

Projektleiter: Hübler, O., Gerlach, K. und Stephan, G.

Beschäftigung zwischen Mobilität und Stabilität

Projektlaufzeit: 6 Jahre (2004-2010)

Projektnummer: P10

Projektleiter: Kraft, $K$.

Auswirkungen organisatorischer Änderungen auf den Unternehmenserfolg

Projektlaufzeit: 6 Jahre (2004-2010)

Projektnummer: P11

Projektleiter: Gehrig-Merz, Monika

Das Arbeitsmarktverhalten von Frauen in Deutschland im Familienkontext: Die Rolle finanzieller Anreize

Projektlaufzeit: 2 Jahre (2004-2006)

Projektnummer: P12

Projektleiter: Möller, J.

Flexibilität der Lohnstruktur, Ungleichheit und Beschäftigung - eine vergleichende Mikrodatenuntersuchung für die USA und Deutschland

Projektlaufzeit: 5 Jahre (2004-2010 mit Unterbrechung)

Projektnummer: P13

Projektleiter: Gürtzgen, N. und Pfeiffer, F.

Lohnhöhe, Rent - Sharing und Tarifbindung
Projektlaufzeit: 6 Jahre (2004-2010)

Projektnummer: P14

Projektleiter: Ronning, G. und Strotmann, $H$.

Auswirkungen von Tarifverträgen und Entgeltöffnungsklauseln auf die Lohnflexibilität und die betriebliche Entwicklung

Projektlaufzeit: 4,5 Jahre (07/2004-12/2008)

Projektnummer: P15

Projektleiter: Schnabel, C. und Wagner, J.

Betriebsgrößenabhängige Schwellenwerte und Arbeitsplatzdynamik in mittelständischen Firmen

Projektlaufzeit: 4 Jahre (2004-2008)

Projektnummer: P16

Projektleiter: Steiner, V.

Arbeitsmarktflexibilisierung durch Subventionierung der Sozialbeiträge im Niedriglohnbereich - Eine Mikrosimulationsstudie für Deutschland

Projektlaufzeit: 6 Jahre (2004-2010)

Projektnummer: P17

Projektleiter: Beblo, M., Wolf, E. und Franz, W.

Quantifizierung der Lohndiskriminierung im Sinne des AGG - Welche Rolle spielen Geschlecht, Alter und Nationalität? Projektlaufzeit: 6 Jahre (10/2004-09/2008 und 01/2009_ 01/2011)

Projektnummer: P18

Projektleiter: Falk, A., Zimmermann, K.F. und Schneider, H. Workfare statt Welfare: Anreizwirkungen und Akzeptanz

Projektlaufzeit: 4,25 Jahre (07/2004-09/2008)

Projektnummer: P19

Projektleiter: Bauer, T., Bender, S. und von Wachter, T.

Betriebliche Lohnbildung und Marktlöhne

Projektlaufzeit: 4 Jahre (2006-2010)

Projektnummer: $P 20$

Projektleiter: Dohmen, T. und Lehmann, H.

In der Black Box: Russische und Ukrainische Firmen und interne Arbeitsmärkte im wirtschaftlichen Umbruch

Projektlaufzeit: 4 Jahre (2006-2010)

Projektnummer: $P 21$

Projektleiter: Haucap, J. und Wey, C.

Endogene Stabilität und endogener Wandel von Arbeitsmarktinstitutionen bei Globalisierung von Arbeits- und Produktmärkten mit unvollständigem Wettbewerb

Projektlaufzeit: 4 Jahre (07/2007-08/2009 und 03/201112/2012)

Projektnummer: $P 22$

Projektleiter: Puhani, $P$.

Arbeitsmarkteffekte sozialpolitischer Maßnahmen

Projektlaufzeit: 4 Jahre (2006-2010)

Projektnummer: P23 
Projektleiter: Riphahn, $R$.

Lohnentwicklung im Lebenszyklus - Eine Analyse von Ausmaß, Begründung und Heterogenität der Lohnsteigerungen für ältere Erwerbstätige

Projektlaufzeit: 3 Jahre (2007-2010)

Projektnummer: P24

Projektleiter: Caliendo, M., Uhlendorff, A.

Suchverhalten bei Arbeitslosigkeit

Projektlaufzeit: 1 Jahr (2008-2009)

Projektnummer: P25

Projektleiter: Spitz-Oener, A.

Flexibilität und Anpassungsfähigkeit Beschäftigter mit Aus-

bildung im Rahmen der dualen Berufsausbildung

Projektlaufzeit: 2,5 Jahre (08/2008-02/2011)

Projektnummer: P26

Projektleiter: Constant, A., Zimmermann, $K$.

Ethnic Diversity and Labor Market Success

Projektlaufzeit: 2 Jahre (09/2009-09/2011)

\section{Anhang B: Internationale Konferenzen und nationale Arbeitstreffen im DFG-Forschungsschwerpunktprogramm}

Das DFG-Forschungsschwerpunktprogramm hat während seiner Laufzeit 2004-2010 sechs internationale Konferenzen (im Jahresrhythmus) und 13 nationale Arbeitstreffen (im Halbjahresrhythmus) veranstaltet. Die offenen Konferenzen und die internen Arbeitstreffen haben die Vernetzung der Arbeitsmarktforscherinnen und -forscher innerhalb und auBerhalb des Forschungsschwerpunktprogramms stark gefördert. Die Konferenzen besetzten zentrale Themen der Forschung im Forschungsschwerpunktprogramm. Es konnten prominente Hauptrednerinnen und -redner für die Konferenzen gewonnen werden.

Im Folgenden beschreiben wir die Themen und Termine der internationalen Konferenzen:

1. "Education and Training: Markets and Institutions"

Ort: ZEW Mannheim

Datum: 18. und 19. März 2005

Hauptredner: David Card and Rick Hanushek

Thema: The purpose of the workshop is to focus upon current empirical research on economic aspects of education and training, including the role of markets and institutions, and to offer policy implications.

2. "Wage Growth and Mobility: Micro-, Macro- and intergenerational Evidence"

Ort: ZEW Mannheim

Datum: 24. und 25. März 2006

Hauptredner: Thomas Lemieux (UBC), Thomas E.
MaCurdy (Stanford), Gary Solon (Michigan)

Thema: The workshop focuses on current empirical research on the determinants of wage growth and mobility over the life cycle and across generations. This includes analysis of life cycle career profiles, the effects of experience and tenure, job search, the time series trends in wages and wage differences, wage changes due to job changes, as well as intergenerational mobility.

3. "Institutions and the Labour Market"

Ort: ZEW Mannheim

Datum: 30. und 31. März 2007

Hauptredner: John DiNardo (University of Michigan), Stephen Machin (University College London), John Pencavel (Stanford University)

Thema: The workshop focuses on theoretical and empirical research of institutions in the labour market. This includes in particular analysis of bargaining, trade unions, employer's associations, works councils, codetermination, judicial decisions concerning industrials actions but also papers to unemployment insurance, in-plant agreements, employment protection, hours' regulations, equal opportunity legislation and workplace organisation are welcome.

4. "Gender and the Labour Market"

Ort: ZEW Mannheim

Datum: 28. und 29. März 2008

Hauptredner: Lena Edlund (Columbia University), Lawrence Kahn (Cornell University), Michael Keane (University of Technology Sydney)

Thema: The workshop focuses on theoretical and empirical research on gender in the labour market. This includes: analysis of gender differences in pre-market human capital, experience, seniority, occupations, earnings, wealth, training and mobility; changing gender roles in changing economies, theories of discrimination; the family as an economic unit; the role of marriage markets; time allocation; policies affecting paid work and family; the impact of welfare reform on single mothers; gender differences in different countries.

5. "Inequality and Poverty in the Global Economy"

Ort: ZEW Mannheim

Datum: 27. und 28. März 2009

Hauptredner: Tony Atkinson (Nuffield College, Oxford), Bruce Meyer (Harris School of Public Policy, University of Chicago)

Thema: Inequality, poverty and social justice are key issues in the policy debate in many countries, with much renewed interest over the last $1 \frac{1 / 2}{2}$ decades. This is due to 
economic internationalisation, increased global competition, the changing influence of labour market institutions and new technologies. This workshop focuses on theoretical and empirical research on inequality, poverty and the labour market. This includes: empirical research documenting changes in inequality and poverty, and how this is related to global trends; analysis of the determinants of inequality and poverty and theoretical work on potential causes; the interplay between poverty and inequality on the one hand, and institutions, anti-poverty policies, technological change and changing demands at the workplace on the other. Further, there is an interest in processes associated with poverty and inequality as well as its causes, like unemployment and gender differences.

6. "Flexibility in Heterogeneous Labour Markets"

\section{Ort: ZEW Mannheim}

Datum: 25.-27. März 2010

Hauptredner: Stephen Machin (University College London/London School of Economics), Jeffrey Smith (University of Michigan), Bruce Weinberg (Ohio State University)

Thema: In today's ever-changing economic conditions, workers and firms are experiencing the constant pressure to adjust employment, wages, working hours, technology, and human capital to meet new needs. Often, workers and firms face the question of whether or not to migrate. Increasing international integration, changing demand for goods and services, as well as changing institutions require labour market flexibility. Government policies to secure the interests of workers and firms are being challenged by changes in goods and labour markets, and increasingly heterogeneous firms and workforces are questioning the efficiency of traditional institutions. This workshop invites empirical and theoretical contributions to this topic from all areas of labour economics and related fields such as the economics of education, industrial economics, health economics, international economics, or public finance.

\section{Literatur}

Akerlof, G.A., Shiller, R.J.: Animal Spirits. How Human Psychology Drives the Economy and why it Matters for Global Capitalism. Princeton University Press, Princeton (2009)

Altonji, J.G., Shakotko, R.A.: Do wages rise with job seniority? Rev. Econ. Stud. 54, 437-459 (1987)

Antonczyk, D., Fitzenberger, B., Sommerfeld, K.: Rising wage inequality, the decline of collective bargaining, and the gender wage gap. Labour Econ. 17(5), 835-847 (2010)

Antonczyk, D., Fitzenberger, B., Sommerfeld, K.: Anstieg der Lohnungleichheit, Rückgang der Tarifbindung und Polarisierung. Z. ArbeitsmarktForschung, in diesem Band (2011)
Arntz, M.: Mobilitätshemmnisse heterogener Arbeitskräfte in Deutschland. Z. ArbeitsmarktForschung, in diesem Band (2011)

Ashenfelter, O., Card, D.: Handbook of Labor Economics, Bd. 4A und 4B. Elsevier, Amsterdam (2011)

Beblo, M., Ohlert, C., Wolf, E.: Logib-D und die Entgeltunterschiede zwischen Männern und Frauen in deutschen Betrieben - Eine Abschätzung des politischen Handlungsfeldes. Z. ArbeitsmarktForschung, in diesem Band (2011)

Beckmann, M., Mühler, G., Schauenberg, B.: Betriebliche Personalpolitik bei heterogenen Arbeitsmärkten. Z. ArbeitsmarktForschung, in diesem Band (2011)

Bellmann, L.: Beschäftigungs- und Lohnwirkungen von betrieblichen Reorganisationsprozessen. Z. ArbeitsmarktForschung, in diesem Band (2011)

Boockmann, B., Steffes, S.: Heterogenität der Beschäftigungsdynamik und Segmentierungsphänomene auf dem deutschen Arbeitsmarkt. Z. ArbeitsmarktForschung, in diesem Band (2011)

Brändle, T., Heinbach, W.D., Maier, M.F.: Tarifliche Öffnung in Deutschland: Ausmaß, Determinanten, Auswirkungen. Z. ArbeitsmarktForschung, in diesem Band (2011)

Brenke, K., Rinne, U., Zimmermann, K.-F.: Kurzarbeit: Nützlich in der Krise, aber nun den Ausstieg einleiten. Wochenbericht des DIW 16, 2-13 (2010)

Caliendo, M., Uhlendorff, A.: Determinanten des Suchverhaltens von Arbeitslosen. Ausgewählte Erkenntnisse basierend auf dem IZA Evaluationsdatensatz. Z. ArbeitsmarktForschung, in diesem Band (2011)

Dustmann, C., Ludsteck, J., Schönberg, U.: Revisiting the German wage structure. Q. J. Econ. 124(2), 843-881 (2009)

Fedorets, A., Spitz-Oener: Flexibilität und Anpassungsfähigkeit Beschäftigter mit Ausbildung im Rahmen der dualen Berufsausbildung. Z. ArbeitsmarktForschung, in diesem Band (2011)

Fitzenberger, B., Hübler, O., Kraft, K.: Flexibilisierungspotenziale bei heterogenen Arbeitsmärkten - Eine Einführung. Z. ArbeitsmarktForschung 41(2-3), 95-116 (2008)

Fitzenberger, B., Hübler, O., Kraft, K.: Arbeitsökonomische Forschung: Theoretisch und empirisch fundiert, ergebnisoffen - Eine Replik auf „Die Mär vom starren deutschen Arbeitsmarkt“. Z. ArbeitsmarktForschung 42(1), 99-106 (2009)

Gerlach, K., Hübler, O., Stephan, G.: Beschäftigung zwischen Mobilität und Stabilität: Empirische Befunde und wirtschaftspolitische Folgerungen. Z. ArbeitsmarktForschung, in diesem Band (2011)

Göddeke, A., Haucap, J., Herr, A., Wey, C.: Stabilität und Wandel von Arbeitsmarktinstitutionen aus wettbewerbstheoretischer Sicht. Z. ArbeitsmarktForschung, in diesem Band (2011)

Gürtzgen, N.: Behindern Flächentarifverträge eine betriebliche Differenzierung der Entlohnung? Z. ArbeitsmarktForschung, in diesem Band (2011)

Heckman, J.: Flexibility and job creation: lessons for Germany. NBER working paper, No. 9194 (2002)

Horn, G.A.: Des Reichtums fette Beute. Wie die Ungleichheit unser Land ruiniert. Campus Verlag, Frankfurt am Main (2011)

Hübler, O.: Safeguarding jobs through labor hoarding in Germanycomment. Appl. Econ. Q., Suppl., 61, 151-165 (2010)

Koller, L., Schnabel, C., Wagner, J.: Beschäftigungswirkungen arbeitsund sozialrechtlicher Schwellenwerte. Z. ArbeitsmarktForschung, in diesem Band (2011)

Konrad, K., Zschäpitz, H.: Schulden ohne Sühne? C.H. Beck, München (2010)

Kraft, K.: Die Auswirkungen von Gewinn- und Entscheidungsbeteiligung auf den Unternehmenserfolg. Z. ArbeitsmarktForschung, in diesem Band (2011) 
Möller, J.: The German labor market response in the world recessionde-mystifying a miracle. Z. ArbeitsmarktForschung 42(4), 325$336(2010)$

Möller, J., König, M.: Lohnungleichheit, Erwerbsbeteiligung und Beschäftigung. Marktkräfte und institutionelle Einflüsse. Z. ArbeitsmarktForschung, in diesem Band (2011)

Müller, K.-U., Steiner, V.: Beschäftigungswirkungen von Lohnsubventionen und Mindestlöhnen - Zur Reform des Niedriglohnsektors in Deutschland. Z. ArbeitsmarktForschung, in diesem Band (2011)

OECD: The OECD Jobs Study: Facts, Analysis, Strategy. OECD, Paris (1994)

OECD: Wage-setting Institutions and Outcomes. Employment Outlook, Chapt. 3. OECD, Paris (2004)

Orlowski, R., Riphahn, R.: Lohnentwicklung im Lebenszyklus Eine Analyse von Ausmaß, Begründung und Heterogenität von Lohnsteigerungen. Z. ArbeitsmarktForschung, in diesem Band (2011)

Puhani, P., Sonderhof, K.: Evaluation sozialpolitischer Reformen. Z. ArbeitsmarktForschung, in diesem Band (2011)

Rinne, U., Schüller, S., Zimmermann, K.-F.: Ethnische Vielfalt und Arbeitsmarkterfolg. Z. ArbeitsmarktForschung, in diesem Band (2011)

Sachverständigenrat [SVR]: Zwanzig Punkte für Beschäftigung und Wachstum. Jahresgutachten 2002/03 des Sachverständigenrates zur Begutachtung der gesamtwirtschaftlichen Entwicklung, Wiesbaden (2002)

Sachverständigenrat [SVR]: Widerstreitende Interessen - Ungenutzte Chancen, Jahresgutachten 2006/07 des Sachverständigenrates zur Begutachtung der gesamtwirtschaftlichen Entwicklung, Wiesbaden (2006)

Sachverständigenrat [SVR]: Das Erreichte nicht verspielen, Jahresgutachten 2007/08 des Sachverständigenrates zur Begutachtung der gesamtwirtschaftlichen Entwicklung, Wiesbaden (2007)

Schneider, H., Uhlendorff, A., Zimmermann, K.-F.: Mit Workfare aus der Sozialhilfe? Lehren aus einem Modellprojekt. Z. ArbeitsmarktForschung, in diesem Band (2011)

Schneider, H., Zimmermann, K.-F.: Agenda 2020: Strategien für eine Politik der Vollbeschäftigung, IZA Standpunkte Nr. 24 (2010)

Sengenberger, W.: Die Mär vom starren deutschen Arbeitsmarkt. Z. ArbeitsmarktForschung 42(1), 93-98 (2009)
Topel, R.: Specific capital, mobility, and wages: wages rise with seniority. J. Polit. Econ. 99(1), 145-176 (1991)

Wilkinson, R., Pickett, K.: The Spirit Level. Why more equal societies almost always do better. Allen Lane, New York (2009)

Bernd Fitzenberger Ph.D. teaches econometrics and labor economics at the University of Freiburg. His research focuses on labor economics and microeconometric methods. Currently, he works on the following topics: wage structure, unions/wage bargaining, duration of unemployment, evaluation of active labor market policies, vocational training, school-to-work transitions, and quantile regression. He is research associate of the ZEW (Mannheim), research fellow at IZA (Bonn), research affiliate at the Institute for Fiscal Studies (London) as well as editor of "Empirical Economics" and member of the editorial board of the "Journal of the European Economic Association" and the "Journal for Labour Market Research". Between 2004 and 2010, he was one of the three coordinators of the DFG Priority Program "Flexibility in heterogeneous labor markets".

Olaf Hübler has taught econometrics at the Leibniz University Hannover. His research focuses on microeconometric analysis in labor economics, especially in the following fields: company-level pacts for employment, short-time work, wage structure, risk attitudes, height and gender in the labor market. He is a research fellow at IAB (Nürnberg) and at IZA (Bonn), member of the editorial board of the "Advances in Statistical Analysis". Between 2004 and 2010, he was one of the three coordinators of the DFG Priority Program "Flexibility in heterogeneous labor markets".

Kornelius Kraft teaches economics at the Technische Universität Dortmund. His research interests are labor economics and industrial organization. Among other topics he investigates the effects of profit sharing, works councils, codetermination, different bargaining structures, management-led firms and the causes of innovative activity. He is a research associate at the ZEW (Mannheim) and a research fellow of the IZA (Bonn). Between 2004 and 2010, he was one of the three coordinators of the DFG Priority Program "Flexibility in heterogeneous labor markets". 